\title{
Optimization of Biplanar Gradient Coils for Magnetic Resonance Imaging
}

\author{
D. Tomasi \\ Medical Department, Brookhaven National Laboratory, 30 Bell Street, Upton, NY, 11973
}

Received on 28 August, 2005; accepted on 28 November, 2005

\begin{abstract}
"Open" magnetic resonance imaging (MRI) scanners are frequently based on electromagnets or permanent magnets, and require self-shielded planar gradient coils to prevent image artifacts resulting from eddy currents in metallic parts of the scanner. This work presents an optimization method for the development of self-shielded gradient coils with biplanar geometry for "Open" MRI scanners. Compared to other optimization methods, this simple approach results in coils that produce larger uniform gradient volumes, and have simple and scalable manufacture.
\end{abstract}

Keywords: Magnetic resonance imaging; MRI scanners

\section{INTRODUCTION}

Open magnetic resonance imaging (MRI) scanners are frequently based on electromagnets or permanent magnets and require planar gradient coils [1]. In these systems, the gap between pole tips is minimized in order to increase the magnetic field strength, $B_{0}$, and the gradient coils need to fit restricted volumes and are located very close to metallic parts of the scanner. This proximity results in eddy currents that cause severe image artifacts, especially for fast imaging modalities such as echo-planar imaging (EPI) that can only be minimized by using self-shielded gradient coils [2-3].

Despite several methods have been developed in the last two decades to improve the quality of the cylindrical gradient coils used in superconductive MRI scanners, and meet the requirements of modern imaging techniques (high magnetic field linearity and gradient efficiency, low coil inductance, and maximal shielding efficiency) [4], limited research has been devoted to the planar geometry [5-7], and the optimization of gradient coils with this geometry is still in its infancy.

Recently I presented the fast simulated annealing (FSA) method, a novel optimization technique for the design of selfshielded gradient coils with cylindrical geometry [8-10]. It combines the simulated annealing (SA) [11-14] and the target field (TF) $[3-4,15]$ techniques to optimize the standard stream functions used to design gradient coils. Compared to standard approaches, this method results in coil with lower inductance that produce larger volumes of gradient field uniformity [9]. Here I present the FSA method for the optimization of selfshielded biplanar gradient coils.

\section{THEORY}

The method described below is proposed for the design of self-shielded gradient sets with biplanar geometry [1, 2]. In these gradient system, currents flow in four parallel $(x, y)$ planes: The primary current density flows in the two inner planes, placed at $z= \pm a$, and the shielding current density flows in the two outer planes, placed at $z= \pm b(a \leq b)$. Currents in the primary and shielding planes must flow in oppo- site sense to null the magnetic field outside the gradient set. For the longitudinal gradient, $G_{z}$, the current density must be anti-symmetric with respect to the $z=0$ plane and have axial symmetry. For the transverse gradient, $G_{x}$, the current distribution must be symmetric with respect to the $z=0$ plane, and invariant along $y$.

Planar stream functions

To take advantage of symmetry conditions we will use the cylindrical $(r, \phi, z)$ and cartesian $(x, y, z)$ frames of references for the longitudinal and transverse gradients, respectively.

Due to the continuity equation $\nabla \cdot \vec{j}=0$, the current densities flowing in the primary planes of the longitudinal and transverse gradients can be obtained by calculating the curl of a vector $\overrightarrow{\mathcal{S}}=(0,0, \mathcal{S})$ : Longitudinal

$$
j_{\phi}(r)=-\frac{d}{d r} \mathcal{S}_{\mathcal{L}}(r), \quad j_{r}(r)=0,
$$

Transverse

$$
j_{y}(x)=-\frac{d}{d x} \mathcal{S}_{\mathcal{T}}(x), \quad j_{x}(x)=0,
$$

where the scalar functions, $\mathcal{S}_{\mathcal{L}}(r)$, and $\mathcal{S}_{\mathcal{T}}(x)$ will be referred as the longitudinal and transverse stream functions, respectively. The spatial dependence of the stream functions can be modeled by using a set of $n$ parameters, $\varepsilon_{i}$. In this work, the following parameterized functions were used:

$S_{\mathcal{L}}(r)=\left\{\begin{array}{lc}\sum_{i=4}^{n} \varepsilon_{i}(r / a)^{i}+\varepsilon_{1} \exp \left\{\varepsilon_{2}\left(r / a-\varepsilon_{3}\right)\right\} & r \leq \varepsilon_{3} a \\ \mathcal{S}_{\mathcal{L}}\left(\varepsilon_{3} a\right) & r>\varepsilon_{3} a\end{array}\right.$

and

$$
\mathcal{S}_{\mathcal{T}}(x)= \begin{cases}\sum_{i=1}^{n} \varepsilon_{i}(x / a)^{i} & |x| \leq l \\ \mathcal{S}_{\mathcal{T}}(l) & |x|>l,\end{cases}
$$




\section{Shielding}

The simplest longitudinal gradient $\left(G_{z}\right)$ coil is the Maxwell arrangement, which is composed by two current loops of radius $R$ carrying opposite currents $I$ in the primary planes. To null the magnetic field produced by this biplanar configuration outside the coil, a current density given by [1]

$$
g_{\phi}(r)=-I R \int_{0}^{\infty} q \frac{\sinh (a q)}{\sinh (b q)}, J_{1}(R q) J_{1}(r q) d q .
$$

is required in the shielding planes, where $J_{1}$ is the Bessel function of order 1. Thus, to shield a continuous current distribution $j_{\phi}\left(r^{\prime}\right)$ flowing in the primary planes of a more general Maxell-like current distribution, a shielding current density

$$
g_{\phi}(r)=-\int_{0}^{\infty} q \frac{\sinh (a q)}{\sinh (b q)} J_{1}(r q) \int_{0}^{\infty} j_{\phi}\left(r^{\prime}\right) J_{1}\left(r^{\prime} q\right) r^{\prime} d r^{\prime} d q,
$$

in the shielding planes is necessary.

The simplest transverse gradient [7] with biplanar geometry consists of two straight wires parallel to the $y$-axis at $x= \pm d$ in the primary planes. To null the magnetic field produced by this distribution outside the coil, a current density

$$
g_{y}(x)=-\frac{I}{\pi} \int_{-\infty}^{\infty} \cos (d q) \cos (x q) \frac{\cosh (a q)}{\cosh (b q)} d q
$$

in the shielding planes is required (see Ref. [1]). Therefore, for a continuous symmetric current density $j_{y}\left(x^{\prime}\right)$ in the primary planes, a shielding current density

$$
g_{y}(x)=-\frac{1}{\pi} \int_{-\infty}^{\infty} \cos (x q) \frac{\cosh (a q)}{\cosh (b q)} \int_{0}^{\infty} j_{y}\left(x^{\prime}\right) \cos \left(x^{\prime} q\right) d x^{\prime} d q
$$

is needed in the shielding planes.

\section{Discrete current distributions}

We used $N$ circular wires with radii $R_{i}(i=1, \cdots, N)$ in each primary plane to make the longitudinal current distribution discrete. The radii were calculated according to:

$$
(i-0.5) I=S_{\mathcal{L}}\left(R_{i}\right)
$$

where $I$ is the current carried by each loop.

Similarly, we used $M$ straight wires in each primary plane at $x_{i}(i=1, \cdots, M)$, which were parallel to the $y$-axis, to make the transverse current distribution discrete. The wire positions $x_{i}$ were calculated according to:

$$
(i-0.5) I=\mathcal{S}_{\mathcal{T}}\left(x_{i}\right)
$$

To make the longitudinal and transverse shielding current densities discrete, similar procedures were used. For the transverse gradient coils, the return path of each wire in the primary planes is a wire in the shielding planes; these coils are similar to rectangular sandwiches formed by two rectangular solenoids of $b-a$ thickness [2]. For inter-connections between the primary and shielding planes, $b-a$ length wires along the $z$-axis were used to null the $z$-component of the magnetic field resulting from these segments. The length of the parallel straight wires was set as $\mathcal{L}>>a$, in order to use the theoretical shielding current density [Eq. (8)].

\section{Simulated annealing}

The stream function parameters were adjusted in order to minimize the dimensionless error function [8],

$$
E=\sum_{i=1}^{\mathcal{N}}\left(1-\frac{G_{i}}{<G>}\right)^{2},
$$

which measures the gradient field dispersion in the region-ofinterest (ROI). The gradient field produced by the gradient coil at a given point of the space, $G$, was calculated at $\mathcal{N}$ points in the ROI [8], by using the Biot-Savart law.

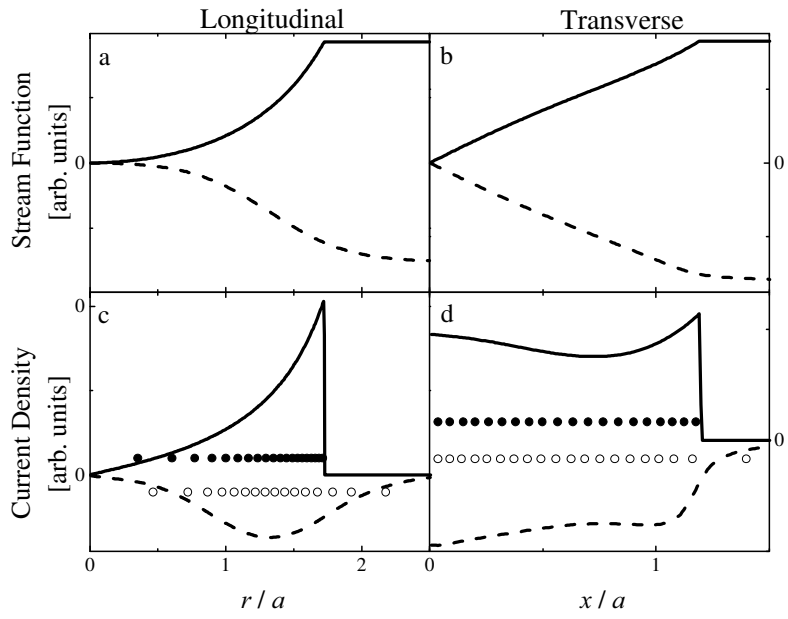

FIG. 1: Optimized stream functions (a and b) and optimal current densities (c and d) as a function of distance for longitudinal (a and c) and transverse (b and d) coils with biplanar geometry. Solid and dashed lines are the optimized primary and shielding distributions, respectively. Solid and open circles are the layouts of the optimal primary and shielding coils, respectively.

\section{RESULTS}

A C-language program, which computes Eq. (1) to (11), was developed to optimize the stream functions. Only 6 adjustable parameters were used for each stream function (Eqs.(3) and (4)). The Biot-Savart calculation of the gradient field [Eq. (11)] was performed over a grid of 16 points, uniformly spaced in a square ROI $(0<x<0.6 a, 0<z<0.6 a)$ in the $y=0$ plane.

The solid lines in Fig. 1 are the optimal stream functions $\mathcal{S}_{\mathcal{L}}(r)$ and $\mathcal{S}_{\mathcal{T}}(x)$ [(a) and (b)], and the resulting current densities $j_{\phi}(r)$ and $j_{y}(x)[(\mathrm{c})$ and (d)] as functions of $r / a$ and $x / a$, respectively; dashed lines in Figs 1a-d are the corresponding shielding distributions. For the longitudinal case $b=1.143 a$ was used, and for the transverse case $b=1.2 a, l=1.257 a$, and $\mathcal{L}=5.714 a$ were used. Figures $\mathrm{c}$ and $\mathrm{d}$ also show the discrete current distributions. For the longitudinal coil, 20 turns in each primary plane and 16 turns in the shielding planes 
TABLE I: Parameters of the optimized stream functions. For longitudinal coil $b=1.143 a$. For transverse coil $b=1.2 a$ and $\mathcal{L}=5.714 a$, and $l=1.257 a$

\begin{tabular}{l|rrrrrrrrr} 
Coil & $\varepsilon_{1}$ & $\varepsilon_{2}$ & $\varepsilon_{3}$ & $\varepsilon_{4}$ & $\varepsilon_{5}$ & $\varepsilon_{6}$ & $\begin{array}{c}\varepsilon_{7} \\
\eta a^{2} \\
{[\mu \mathrm{T} \mathrm{m} / \mathrm{A}]}\end{array}$ & $\begin{array}{c}L / a \\
{[\mathrm{mH} / \mathrm{m}]}\end{array}$ \\
\hline $\begin{array}{l}\text { Longitudinal } \\
\text { Transverse }\end{array}$ & 0.850 & 4.357 & 1.740 & 0.000 & 0.913 & 0.000 & 0.183 & 3.246 & 0.989 \\
\hline
\end{tabular}

TABLE II: Magnetic field and electrical properties corresponding to shielded biplanar gradient coils. $\mathrm{N}=18$ (36) copper wires with $0.5 \mathrm{~mm}$ diameter were used in each primary plane, for longitudinal (transverse) coils. $a=3.5 \mathrm{~cm}$.

\begin{tabular}{|c|c|c|c|c|c|c|c|c|}
\hline Coil & & $\begin{array}{c}b \\
{[\mathrm{~cm}]}\end{array}$ & $\begin{array}{c}z-\mathrm{HGV} \\
{[\mathrm{cm}]}\end{array}$ & $\begin{array}{c}x \text {-HGV } \\
{[\mathrm{cm}]}\end{array}$ & $\begin{array}{c}\eta \\
{[\mathrm{mT} / \mathrm{m} / \mathrm{A}]}\end{array}$ & $\begin{array}{c}L \\
{[\mu \mathrm{H}]}\end{array}$ & $\begin{array}{c}\mathrm{R} \\
{[\Omega]}\end{array}$ & $\begin{array}{c}\kappa \\
{[\%]}\end{array}$ \\
\hline \multicolumn{9}{|c|}{ 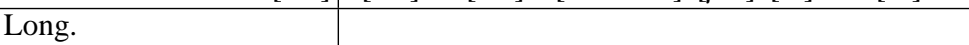 } \\
\hline & Maxwell pair & NS & 3.08 & 3.84 & 8.88 & 186 & 0.8 & Unshielded \\
\hline & $3^{\text {rd }}$ Order [1] & 4.15 & 2.14 & 3.24 & 4.64 & 157 & 1.0 & 91.56 \\
\hline & Ref. [2] & 4.00 & 5.16 & 4.22 & 2.12 & 32 & 1.3 & 95.26 \\
\hline & This work & 4.00 & 5.08 & 5.28 & 2.45 & 28 & 1.5 & 91.44 \\
\hline \multicolumn{9}{|c|}{ Tran. } \\
\hline & Ref. [7] & 4.15 & 2.25 & 2.50 & 2.13 & 280 & 2.5 & 76.00 \\
\hline & $3^{\text {rd }}$ Order [1] & 4.20 & 1.84 & 2.16 & 2.61 & 279 & 2.5 & 96.40 \\
\hline & $5^{\text {th }}$ Order [1] & 4.20 & 3.08 & 4.20 & 0.82 & 187 & 2.5 & 90.70 \\
\hline & Ref. [2] & 4.15 & 4.20 & 4.20 & 0.89 & 60 & 2.5 & 92.00 \\
\hline & This work & 4.15 & 4.44 & 4.64 & 0.81 & 49 & 2.5 & 91.00 \\
\hline
\end{tabular}

were used. For the transverse coil 40 turns were used in each plane.

The optimized stream function parameters are listed in Table 1 ; this table also lists the gradient efficiency at the gradient isocenter, $\eta$, and coil inductance, $L$, corresponding to the coils in Fig 1 . Since $\eta$ decreases quadraticaly, and $L$ increases linearly with $a$, values in Table 1 are generalized as $\eta a^{2}$ and $L / a$. For the longitudinal coil, $L$ was evaluated by using [2]

$$
L=4 \pi \mu_{0} \int_{0}^{\infty}\left|\sum_{i=1}^{n} R_{i} J_{1}\left(R_{i} q\right)\right|^{2}\left(1-\frac{\sinh (a q)}{\sinh (b q)} e^{-(b-a) q}\right) \sinh (a q) e^{-a q} d q
$$

and for the transverse coil [2]

$$
L=\frac{8 \mathcal{L} \mu_{0}}{\pi} \int_{0}^{\infty}\left|\sum_{i=1}^{n} \cos \left(x_{i} q\right)\right|^{2}\left(1-\frac{\cosh (a q)}{\cosh (b q)} e^{-(b-a) q}\right) \frac{\cosh (a q)}{q} e^{-a q} d q
$$

was used. Here $R_{i}$ and $x_{i}$ are the positions of the $i$-wire in the longitudinal and transverse coils, respectively. These expressions includes self and mutual inductance between the wires in the primary and shielding planes.

Figure 2 shows the efficiency of the shielding coil to null the magnetic field in the outer region. These Biot-Savart calculations were performed for $a=0.035 \mathrm{~m}$ and $z=0.05 \mathrm{~m}$. Solid lines are the $z$-component of the magnetic field produced by primary coils and dashed lines correspond to the field produced by both primary and shielding coils in Fig 1. As shown in these figures, the shielding coils cancel at least $95 \%$ of the unshielded fields at this axial position $(z=1.423 a)$, which corresponds to the position of the pole tips. The curves in Fig. 3 are contour plots showing the limits of the 95\% homogeneous gradient volume (HGV) produced by the proposed gradient coils; solid and dashed lines correspond to the coils in Fig. 1. Coils designed by this method have very large HGV. For the longitudinal gradient, the $\mathrm{HGV}$ has a spherical radius of $0.75 a$. For the transverse gradient, the $\mathrm{HGV}$ is a cylinder coaxial to the $z$-axis with height $=2.6 a$, and radius $=0.65 a$. The calculated values for the extension of the $95 \% \mathrm{HGV}$ along the $z-$ and $x$-axes, the gradient efficiency, coil inductance and resistance, $L$ and $R$, and the overall shielding efficiency, $\kappa$, corresponding to the coils in Figs 4 and 5 are listed in Table 


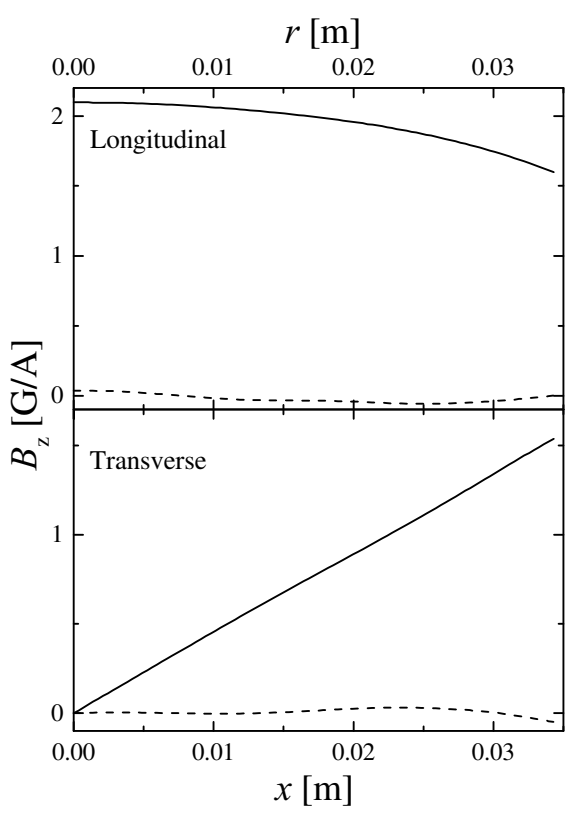

FIG. 2: Shielding performance as a function of distance, for the longitudinal (top panel) and transverse (bottom panel) gradient coils. The curves are Biot-Savart calculations of the $z$-component of the magnetic field produced by the unshielded (primary plane; solid line), and shielded (primary and shielding planes; dashed lines) at $z=5 \mathrm{~cm}$.

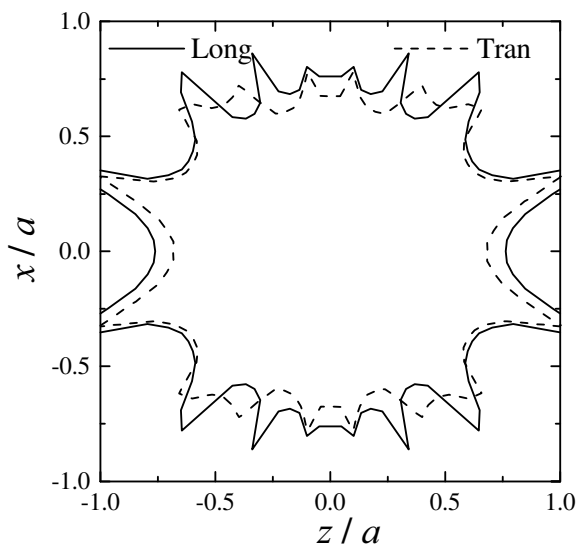

FIG. 3: Contours of the $95 \%$ HGV produced by the gradient coils in the coronal plane. The solid (dashed) line corresponds to the longitudinal (transverse) design.

\section{2 , for $a=3.5 \mathrm{~cm}$}

The upper panels of Figs. 4 and 5 compare the layouts of the primary (solid circles) and shielding (solid triangles) coils proposed in Ref. [2] for the longitudinal and transverse gradients, respectively, with those resulting from this method (open symbols). The optimized primary (solid line) and shielding (dashed line) stream functions used to get the wire positions are also shown in these figures. One advantage of the proposed method is related to the coil fabrication process. The coil layout corresponding to Ref. [2] is more difficult to accomplish because of the shorter minimal distance between neighbor wires. The present method uses smooth stream functions, which result in larger minimal gap between neighbor wires, facilitating coil construction.

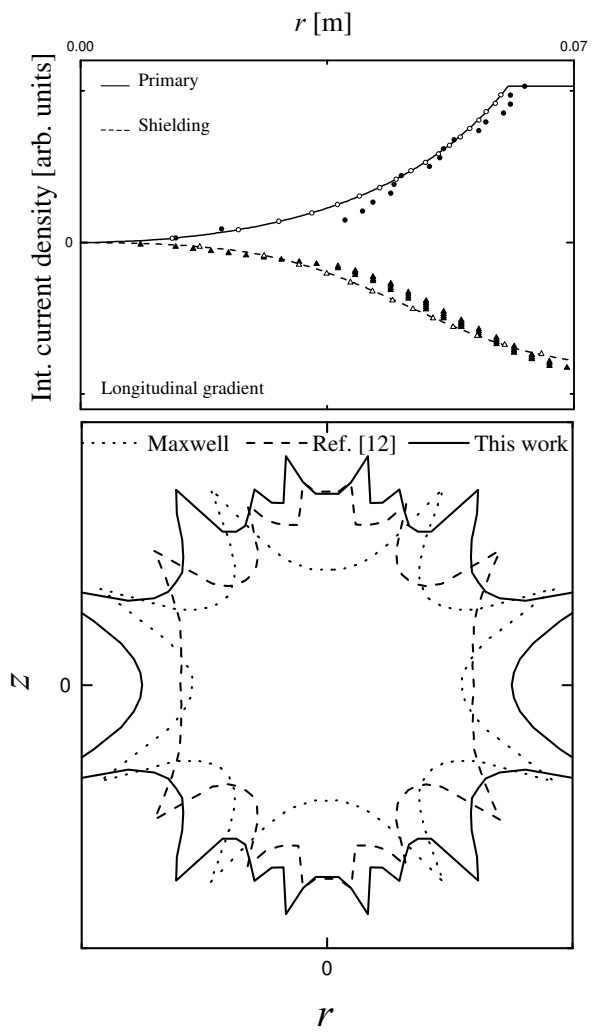

FIG. 4: [Top] Comparison of coil layouts resulting from the fast simulated annealing method [2], and this work for the longitudinal gradient coil. The solid circles (triangles) are the wire positions in the primary (shielding) plane of ref [2]. The solid (dashed) line is the optimal stream function, and the open circles (triangles) are the wire positions in the primary (shielding) plane. [Bottom] Contours of the 95\% HGV produced by the gradient coils in the coronal plane.

The bottom panels of Figs. 4 and 5 compare the limits of the 95\% HGV produced by longitudinal and transverse gradient coils, respectively, in the coronal plane. Solid, dashed, and dotted lines correspond to the designs achieved in this work, Ref. [2], and Ref. [7], respectively. As shown in these figures, the optimization of stream functions results in coils producing larger HGV than those designed by our previous method [2].

\section{CONCLUSION}

This work presents a method for the development of gradient coils with planar geometry, which is based on the numerical optimization of biplanar stream functions. This method allows for the design of self-shielded, low-inductance gradient coils. The coils produce larger uniform gradient volumes than those resulting from previous optimization methods. Furthermore, the designs can be scaled and their simplicity makes possible easier coil manufacture. 

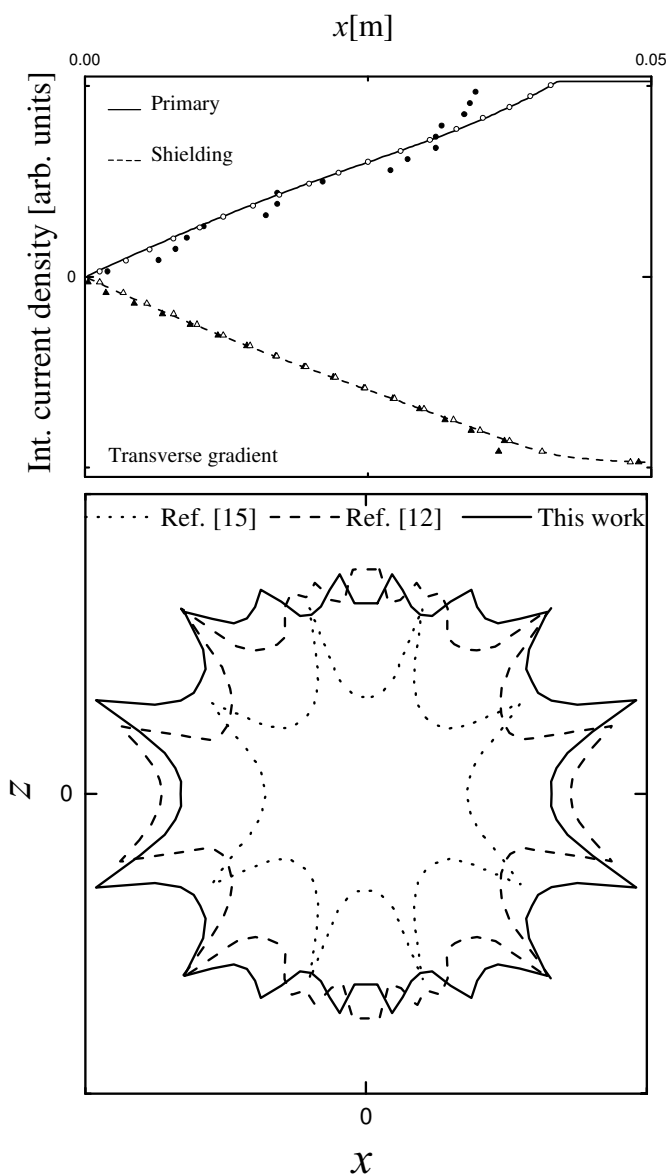

FIG. 5: [Top] Comparison of coil layouts resulting from the fast simulated annealing method [2], and this work for the transverse gradient coil. The solid circles (triangles) are the wire positions in the primary (shielding) plane of ref [2]. The solid (dashed) lines is the optimal stream function, and the open circles (triangles) are the wire positions for the primary (shielding) plane in this work. [Bottom] Contours of the 95\% HGV produced by the gradient coils in the coronal plane.

[1] E. C. Caparelli, D. Tomasi, and H. Panepucci, J. Magn. Reson. Imaging 9, 725 (1999)

[2] D. Tomasi, E. C. Caparelli, H. Panepucci, and B. Foerster, Set. J. Magn. Reson. 140, 325 (1999).

[3] K. Yoda, J. Appl. Phys. 67, 4349 (1990).

[4] R. Turner, Magn. Reson. Imaging. 11, 903 (1993).

[5] R. Bowtell, P. Mansfield, Minimum power, flat gradient pairs for NMR Microscopy - 8th ISMRM 1990.

[6] A. M. Peters, R. W. Bowtell, Magn. Reson. Materials in Physics, Biology, and Medicine, 2, 387 (1994).

[7] V. Bangert, P. Mansfield, J. Phys. E: Sci Instrum. 15, 235 (1982).

[8] D. Tomasi, Magn. Reson. Med. 45, 505 (2001).

[9] D. Tomasi. FSA design of a gradient coil set for head imag- ing. Proceedings of the IV Mexican Symposium on Medical Physics, Juriquilla, Queretaro, México, 2001. AIP Conference Proceedings 593, 1 (2001).

[10] D. Tomasi, R.F. Xavier, B. Foerster, H. Panepucci, A. Tannús, and E.L. Vidoto. Magn. Reson. Med. 48, 707 (2002).

[11] S. Crozier, D. M. Doddrell, J. Magn. Reson. A 103, 354 (1993).

[12] S. Crozier, L. K. Forbes, and D. M. Doddrell, J. Magn. Reson. A107, 126 (1994).

[13] S. Crozier, D. M. Doddrell, Magn. Reson. Imaging 13, 615 (1995).

[14] M. L. Buszko, M. F. Kempka, E. Szczesniak, D. C. Wang, and E. R. Andrew, J. Magn. Reson. B 112, 207 (1996).

[15] B. A. Chronik, B. K. Rutt, Magn. Reson. Med. 39, 270 (1998). 